\title{
IN VITRO AND IN VIVO BIOCOMPATIBILITY STUDIES OF ZNO NANOPARTICLES
}

\author{
YUFENG ZHENG and RANZHONG LI \\ Department of Advanced Materials and Nanotechnology, College of Engineering, Peking University, \\ No. 5 Yi-He-Yuan Road, Beijing 100871, China \\ yfzheng@pku.edu.cn,lsk455@163.com \\ YUEDAN WANG \\ Department of Immunology, School of Basic Medicine, Peking University, \\ No. 38, Xue-Yuan Road, Beijing 100083, China \\ wangyuedan@bjmu.edu.cn
}

\begin{abstract}
The in vitro and in vivo biocompatibility of the $\mathrm{ZnO}$ nanoparticles were evaluated using cell model and animal model, by MTT assay, flow cell cytometry, pathological optical and electron microscopy examinations. Both the L929 cell and Hela cell proliferative activity were strongly inhibited by the presence of $\mathrm{ZnO}$ nanoparticles, no matter cultured with low dose and high dose suspension. AnnexinV-FITC/PI-FCM assay showed that the number of necrotic cell and apoptotic cell increased significantly. Feeding the $\mathrm{ZnO}$ nanoparticle suspension through digestive tract would lead to the damage to some primary organs (heart, lung, liver and kidney). Further investigation by TEM showed the expanded sarcoplasmic reticulum and organelle vacuolation features.
\end{abstract}

Keywords: $\mathrm{ZnO}$; biocompatibility; cell proliferation; apoptosis.

\section{Introduction}

Being a key functional material with versatile properties, such as dual semiconducting and piezoelectric properties, $\mathrm{ZnO}$ has important applications in optoelectronic devices, sensors, lasers, transducers, and photovoltaic devices[1-2]. ZnO nanoparticles have been used in many applications in our daily life, such as drug carriers and cosmetics[3]. However, although inhalation of ultrafine $\mathrm{ZnO}$ particles at relatively high dose (500 $\mathrm{mg} / \mathrm{m} 3$ ) for 2 hours did not induce acute systemic effects in humans, inhalation of $\mathrm{ZnO}$ fumes in an occupational setting can cause metal fume fever (fatigue, chills, fever, myalgias, cough, dyspnea, leukocytosis, metallic taste, and salivation)[4]. The interaction of $\mathrm{ZnO}$ nanomaterial with the organism is still far known from the viewpoint of biomaterial scientist. The objective of the present study is to evaluate both the in vitro and in vivo biocompatibility of the $\mathrm{ZnO}$ nanoparticles using cell model (both mouse fibroblast and human cervical cancer cells) and animal model (ICR mouse).

\section{Experimental Procedure}

$\mathrm{ZnO}$, with mean diameter 20nm, as identified by TEM and HREM in Fig. 1, were made by Shanghai Huijing Sub-Nano scale New Material Company, LTD. The ZnO 
nanoparticles was distributed into RPMI 1640 culture medium containing $10 \%$ fetal bovine serum (FBS) to become $400 \mu \mathrm{g} / \mathrm{ml}$ suspension, then were high temperature \& pressure sterilized and ultrasonically vibrated for 10 minutes before use.

L929 and Hela cells were cultured in RPMI1640 supplemented with 10\% fetal bovine serum and 1\% L-glutamine, penicillin, and streptomycin. The MTT (3-(4,5-dimethyl-2thiazolyl)-2,5-diphenyl-2H-tetrazolium bromide) assay was used to evaluate proliferative activity. Exposure to $50 \mu \mathrm{l}$ RPMI1640 was used as a negative control and $50 \mu \mathrm{DMSO}$ as a positive control. At least three time points are depicted for each assay.

30 ICR mice, male, aged 6-8 weeks and weighing 18-25g, were used in the experiment, with 15 each for control group and $\mathrm{ZnO}(20 \mathrm{~nm})$ group. The $30 \mathrm{mg} / \mathrm{ml}$ suspension was administered at a dose of $0.2 \mathrm{ml}$ through digestive tract, each time a day and lasted 7 days. The animals were observed if they were illed or died during the experiment, and at day 17 were killed by exsanguinations following pelltobarbitalum natricum anaesthesia. The blood was conserved by adding heparin. All animals were examined grossly. Tissues including liver, kidney, heart, spleen, lung and brain were collected. Moiety were fixed in a $10 \%$ formaldehyde solution and others were freezed in $-20^{\circ} \mathrm{C}$. After fixation, tissues were routinely processed, embedded in paraffin, cut at a microtome setting of $5 \mu \mathrm{m}$, mounted on glass slides, stained with hematoxylin and eosin and examined by light microscopy. After dehydration and resin embedding, some tissues were sectioned to 70nm thick, stained with uranyl acetate and lead citrate and examined by JEM-100 CX II transmission electron microscopy. The animal experiment was performed in accordance with the Guide for the Care and Use of Laboratory Animals, with the approval of the Beijing Municipal Science and Technology Committee.
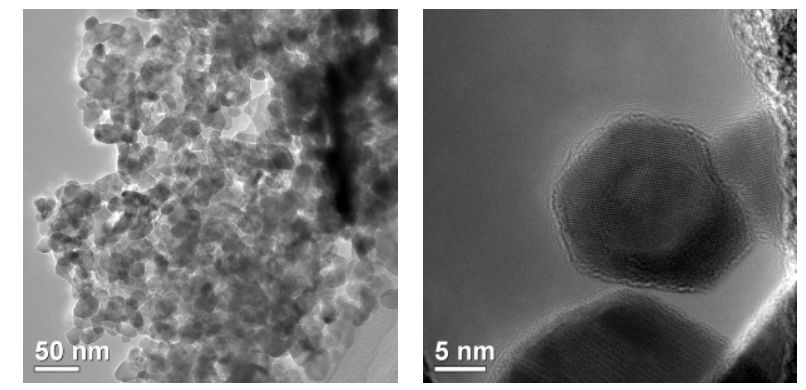

Fig. 1. TEM bright image and HREM image of the $\mathrm{ZnO}$ nanoparticles.

\section{Results and Discussion}

\subsection{In vitro biocompatibility evaluation of $\mathrm{ZnO}$ nanoparticles}

Table 1 and Table 2 listed the optical density (OD) and relative growth rate (RGR) of L929 cells and Hela cells based on the results of MTT assay. It can be seen that both the L929 cell and Hela cell proliferative activity were strongly inhibited by the presence of nano $\mathrm{ZnO}$, no matter at low dose and high dose. The RGR values were quite different 
from the negative control (normal cell) and was evidently similar to the positive control $(\mathrm{DMSO})(\mathrm{p}<0.05)$, which indicates the heavy cytotoxicity of $\mathrm{ZnO}$ nanoparticles.

Table 1. The optical density (OD) and relative growth rate (RGR) of L929 cells based on the results of MTT assay.

\begin{tabular}{llllll}
\hline \multirow{2}{*}{ Group/Concentration } & & $24 \mathrm{~h}$ & & $48 \mathrm{~h}$ & \\
& & High & Low & High & Low \\
\hline \multirow{2}{*}{ Normal L929 cell } & OD & 0.3909 & & 0.9446 & \\
& RGR & $100 \%$ & & $100 \%$ & \\
\multirow{2}{*}{ ZnO (20nm) } & OD & 0.0345 & 0.0237 & 0.0430 & 0.0400 \\
& RGR & $8.83 \%$ & $6.07 \%$ & $4.55 \%$ & $4.23 \%$ \\
\multirow{2}{*}{$12 \%$ DMSO } & OD & 0.030 & & 0.017 & \\
& RGR & $7.674 \%$ & & $1.78 \%$ & \\
\hline
\end{tabular}

Table 2. The optical density (OD) and relative growth rate (RGR) of Hela cells based on the results of MTT assay.

\begin{tabular}{llllll}
\hline Group/Concentration & & $24 \mathrm{~h}$ & & $48 \mathrm{~h}$ & \\
& & High & Low & High & Low \\
\hline \multirow{2}{*}{ Normal Hela cell } & OD & 0.5839 & & 0.774 & \\
& RGR & $100 \%$ & & $100 \%$ & \\
\multirow{2}{*}{$\mathrm{ZnO}(20 \mathrm{~nm})$} & OD & 0.0871 & 0.1022 & 0.107 & 0.118 \\
& RGR & $14.91 \%$ & $17.51 \%$ & $13.82 \%$ & $15.24 \%$ \\
\multirow{2}{*}{$12 \%$ DMSO } & OD & 0.046 & & 0.090 & \\
& RGR & $7.88 \%$ & & $11.63 \%$ & \\
\hline
\end{tabular}

Figures 2 and 3 illustrated the early L929 cell and Hela cell apoptosis percentages detected by AnnexinV-FITC/PI -FCM assay at $24 \mathrm{~h}$ and $48 \mathrm{~h}$. The dot lied in the lower left quadrant, lower right quadrant, upper right quadrant and upper left quadrant represents the living cell, apoptotic cell, secondary necrotic cell and mechanical destruction of cell during culture procedure, respectively. For $\mathrm{ZnO}$ nanoparticle group, the percentage of necrotic cell is the dominated for both the L929 cell and Hela cell, with over $60 \%$ at $24 \mathrm{~h}$ and over $80 \%$ at $48 \mathrm{~h}$. The following is the percentage of the apoptotic cell. Figure 4 took the Hela cell after cultured in $\mathrm{ZnO}(20 \mathrm{~nm})$ suspension for $24 \mathrm{~h}$ as example to show the typical morphologies under TEM, and both condensed new moontype chromatin and irregular organelle morphology are observed.

\subsection{In vivo biocompatibility evaluation of $\mathrm{ZnO}$ nanoparticles}

Figure 5 showed the light optical image of the mouse organs including heart, lung, liver, spleen, kidney and brain. The spleen and brain cells are normal in comparison to the blank group, whereas the heart, lung, liver and kidney cells turned abnormal. Cytoplasmic coagulation and inflammatory cell infiltration happened in the heart, few lymphocyte infiltration and broadened alveolar septum could be found in the lung, hydropic degeneration could be observed in the liver, and glomerular swelling and tubular swelling was obvious in the kidney. 
Figure 6 illustrated the TEM images of the cardiac tissue(a)-(b), liver tissue(c)-(d) and brain tissue(e)-(f) of the experimental mouse. Expanded sarcoplasmic reticulum and vacuolar degeneration could be found in the cardiac tissue, as shown in Fig. 6(a) and Fig. 6(b). Organelle vacuolation is the typical feature of the liver tissue, as indicated in Fig. 6(c) and Fig. 6(d). Loose axon, organelle vacuolation and vacuolar degeneration could be observed inside the brain tissue, as illustrated in Fig. 6(e) and Fig. 6(f).

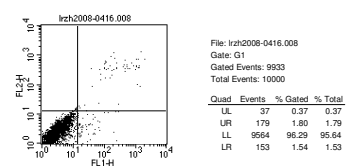

(a)
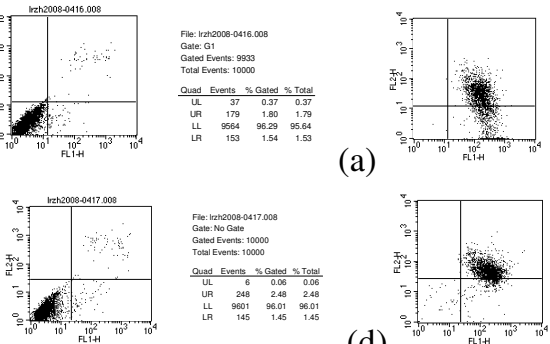

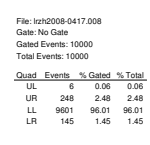

(d)

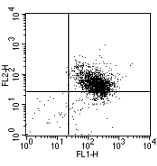

(b)
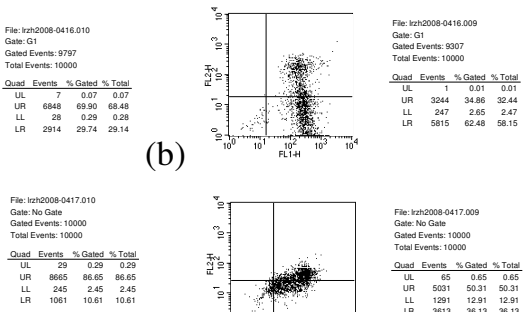

(e)

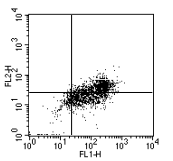

(c)

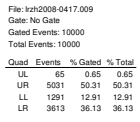

(f)

Fig. 2. AnnexinV-FITC/PI -FCM assay on L929 cell. (a) blank control, 24h; (b) ZnO (20nm), 24h; (c) DMSO, 24h; (d) blank control, 48h; (e) $\mathrm{ZnO}$ (20nm), 48h; (f) DMSO, 48h.
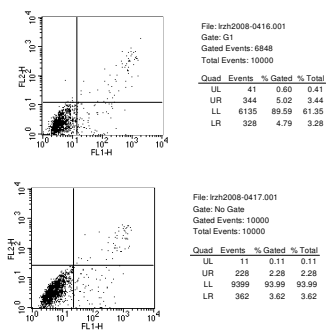

(d)
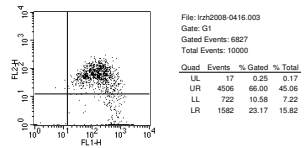

(a)
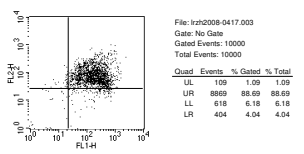

(e)
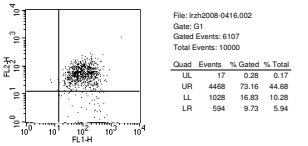

(b)

(c)
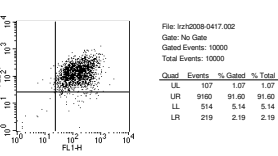

(f)

Fig. 3. AnnexinV-FITC/PI -FCM assay on Hela cell. (a) blank control, 24h; (b) ZnO (20nm), 24h; (c) DMSO, 24h; (d) blank control, 48h; (e) $\mathrm{ZnO}$ (20nm), 48h; (f) DMSO, 48h.
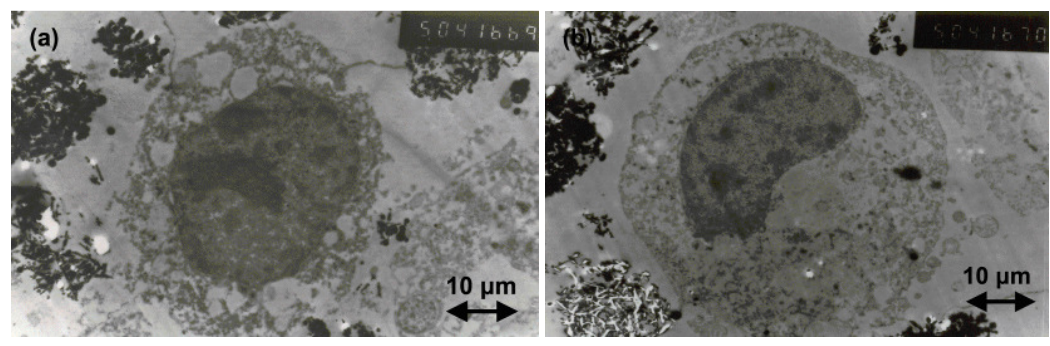

Fig. 4. TEM image of Hela cell after cultured in $\mathrm{ZnO}(20 \mathrm{~nm})$ suspension for $24 \mathrm{~h}$ showing the typical morphologies (a) condensed new moon-type chromatin and (b) irregular organelle morphology. 

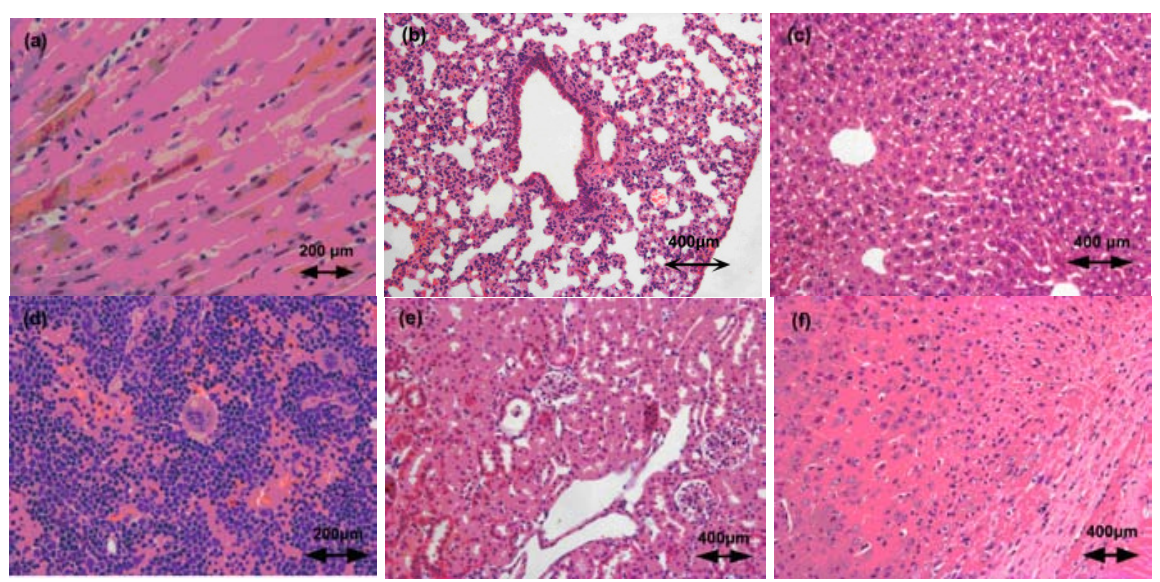

Fig. 5. Light microscopic observation of the organ of the mouse after feeding $\mathrm{ZnO}(20 \mathrm{~nm})$ nanoparticle suspension (a) heart; (b), lung; (c) liver; (d) spleen; (e) kidney; (f) brain.

Accurately assessing the toxicity and safety of $\mathrm{ZnO}$ nanomaterials is of utmost importance. On the one hand, the present work shows that the $\mathrm{ZnO}$ nanoparticles with the diameter exhibit toxicity both in vitro and in vivo, and it suggests that the further application of $\mathrm{ZnO}$ nanoparticles in the body fluid should be cautious. Dechsakulthorn et al.[5] assessed the cytotoxicity of $\mathrm{ZnO}$ nanopowder with particle size $50-70 \mathrm{~nm}$ in human skin fibroblasts using the colourimetric MTS (3-(4,5-dimethylthiazol-2-yl)-5-(3carboxymethoxyphenyl)-2-(4-sulfophenyl)-2Htetrazolium) in vitro assay, and his work indicated higher toxicity of $\mathrm{ZnO}$ at both 4 and $24 \mathrm{~h}$. Lin et al.[6] had also demonstrated that $70 \mathrm{~nm}$ and $420 \mathrm{~nm} \mathrm{ZnO}$ particles significantly reduce cell viability and cause oxidative DNA damage in a dose- and time dependent manner in A549 cells. On the other hand, recent study by Hanley[7] found that $\mathrm{ZnO}$ nanoparticles have intrinsic properties that enable them to preferentially kill cancerous T-cells while sparing healthy normal cells. The preferential toxicity of $\mathrm{ZnO}$ nanoparticles towards cancerous $\mathrm{T}$-cells is of substantial magnitude ( 28-35 times), especially in comparison with ex vivo indices of $\leq 10$ reported for other commonly used chemotherapeutic agents using similar assays. This inherent differential toxicity of $\mathrm{ZnO}$ nanoparticles against rapidly dividing cancer cells raises exciting opportunities for their potential use as anti-cancer agents.

\section{Conclusion}

(1) Both the L929 cell and Hela cell proliferative activity were strongly deteriorated by different dose of $20 \mathrm{~nm} \mathrm{ZnO}$ nanoparticle suspension at $24 \mathrm{~h}$ and $48 \mathrm{~h}$ culture.

(2) The percentage of necrotic L929/Hela cell and the apoptotic L929/Hela cell are absolute majority in AnnexinV-FITC/PI-FCM assay.

(3) Feeding the $\mathrm{ZnO}$ nanoparticle suspension through digestive tract at a dose of $0.6 \mathrm{mg}$ daily led to the damage to some primary organs (heart, lung, liver and kidney) of mice. 
(4) Sarcoplasmic reticulum expanded and organelle vacuolated in the mice heart, liver and brain, after feeding the $\mathrm{ZnO}$ nanoparticle suspension through digestive tract.

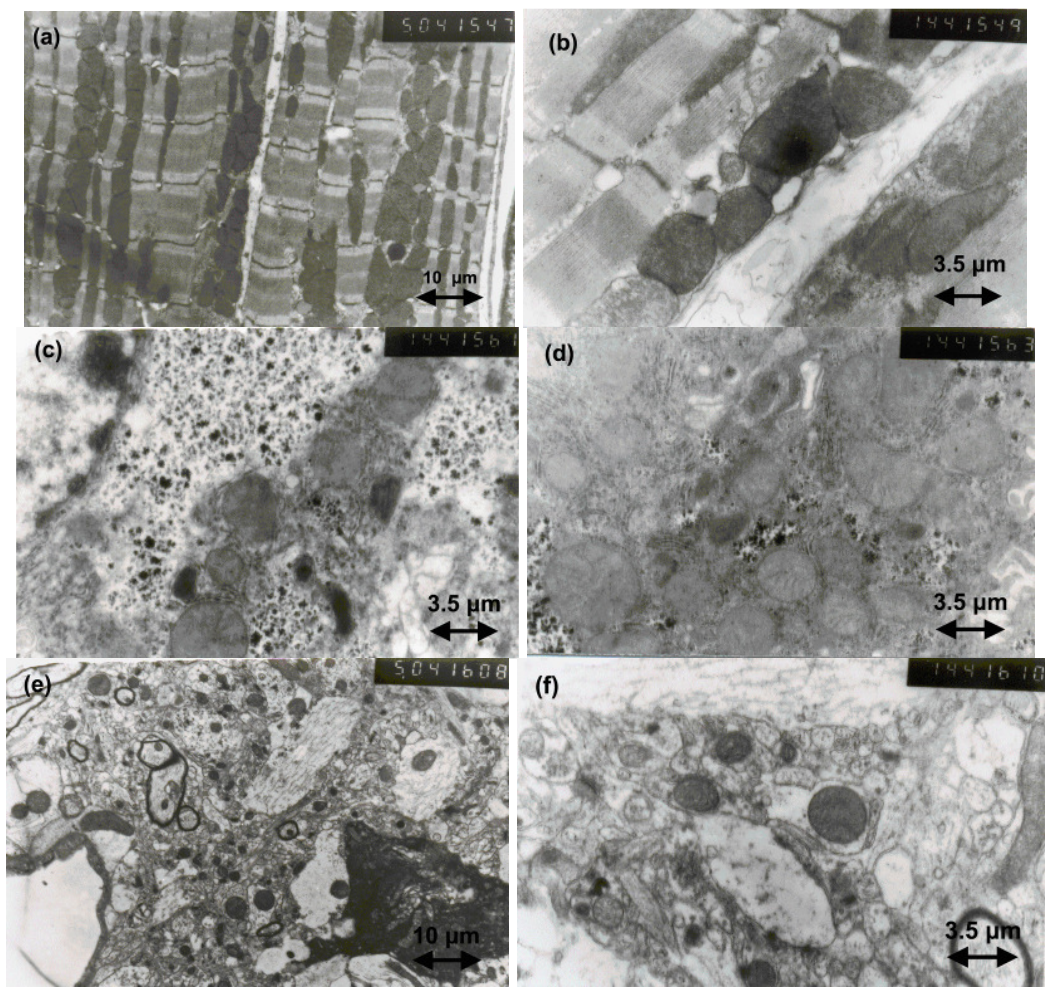

Fig. 6. TEM images of the mouse cardiac tissue(a)-(b), liver tissue(c)-(d) and brain tissue(e)-(f) after the $30 \mathrm{mg} / \mathrm{ml}$ nano $\mathrm{ZnO}$ suspension was administered at a dose of $0.2 \mathrm{ml}$ through digestive tract, each time a day and lasted 7 days, then executed at the day 17 .

\section{References}

1. Z. W. Pan, Z. R. Dai, Z. L. Wang, Science 291, 1947 (2001).

2. Z. L. Wang, X. Y. Kong, Y. Ding, P. X. Gao, W. Hughes, R. S. Yang, Y. Zhang, Adv. Funct. Mater. 14, 944 (2004).

3. J. Zhou, N. S. Xu, Z. L. Wang, Adv. Mater. 18, 2432, (2006).

4. W. S. Beckett, D. F. Chalupa, A. Pauly-Brown, D. M. Speers, J. C. Stewart, M. W. Frampton, M. J. Utell, Li-Shan Huang, C. Cox, W. Zareba and G. Oberdörster, American Journal of Respiratory and Critical Care Medicine 171, 1129 (2005).

5. F. Dechsakulthorn, A. Hayes, S. Bakand, L. Joeng and C. Winder, in Proc. 6th World Congress on Alternatives \& Animal Use in the Life Sciences, August 21-25, 2007, Tokyo, Japan, AATEX 14, Special Issue, pp. 397-400.

6. W. S. Lin, Y. Xu, C. C. Huang, Y. F. Ma, K. B. Shannon, D. R. Chen, Y. W. Huang, J. Nanopart. Res., (2008) DOI 10.1007/s11051-008-9419-7.

7. C. Hanley, J. Layne, A. Punnoose, K. M. Reddy, I. Coombs, A. Coombs, K. Feris and D. Wingett, Nanotechnology 19, 295103 (2008). 